\title{
Using an International Gaming Tournament to Study Individual Differences in MOBA Expertise and Cognitive Skills
}

\author{
Justin W. Bonny \\ foundry 10 \\ Seattle, WA USA \\ justin@foundry10.org
}

\author{
Lisa M. Castaneda \\ foundry 10 \\ Seattle, WA USA \\ lisa@foundry10.org
}

\author{
Tom Swanson \\ foundry10 \\ Seattle, WA USA \\ tom@foundry10.org
}

\begin{abstract}
In this study we evaluated a novel approach for examining the link between gaming expertise and cognitive skills, and the value of recruiting and running participants at a MOBA gaming tournament. Participants completed a set of cognitive tasks that measured spatial working and long term (location) memory, basic cognitive processing, and gaming experience. Comparable reliability on the working memory task and results in line with previous research on the location memory task indicated the data collected was valid and reliable. We observed a significant relation between gaming experience and response time on the location memory task. We discuss that conducting gaming research at a tournament is a valid way of collecting data for a gaming expertise study while providing a range of gaming expertise that may not be available when recruiting at college campuses. Furthermore, our results extend previous gaming research that suggests that individual differences in gaming experience are correlated with the speed of recalling spatial information from long term memory.
\end{abstract}

\section{Author Keywords}

Gaming expertise; individual differences; MOBA; cognitive skills; working memory; spatial memory

\section{INTRODUCTION}

The continued rise of video game play among individuals has increased interest in the impact of video game experience on cognitive skills. Video game play has grown in popularity in recent years with research showing $97 \%$ of teens and $81 \%$ of American adults ages 18-29 have played video games [25]. Alongside the rise in popularity of these competitive games is a growing body of research examining whether individual differences in cognitive skills are linked to gaming experience and expertise. There is evidence that video gamers outperform non-gamers on measures of attention and spatial perception $[3,19]$, processing speed [32], and, in some studies, working memory $[11,32]$. As the majority of this

Permission to make digital or hard copies of part or all of this work for personal or classroom use is granted without fee provided that copies are not made or distributed for profit or commercial advantage and that copies bear this notice and the full citation on the first page. Copyrights for third-party components of this work must be honored. For all other uses, contact the Owner/Author. Copyright is held by the owner/author(s).

CHI'16, May 07-12, 2016, San Jose, CA, USA

ACM 978-1-4503-3362-7/16/05.

http://dx.doi.org/10.1145/2858036.2858190 research has focused on college students who play first person shooter (FPS) games, an open question is whether individual differences in other gaming experiences are also connected to cognitive skills.

A specific type of video game experience, multiplayer online battle arena or MOBA, has undergone particularly rapid growth in players and viewers. Typically, in these games, players each select a unique avatar (often called a hero) and are sorted into two teams of five which compete to destroy their opponent's headquarters. In addition to being played by casual gamers, MOBAs have also been used as an eSport with matches where expert gamers compete for cash prizes while a live audience views the gameplay. Similar to professional sports, millions of viewers watch video game matches with recent MOBA tournaments attracting more viewers than NBA finals games [7,20,41]. Since MOBA players have a wide range in expertise, from casual to professional gamers, they offer a unique opportunity to explore whether gamers of varying expertise levels also vary in their cognitive skills. In addressing this question we also aim to address concerns that have been raised in regard to the small sample sizes and lack of a range of expertise levels that have been argued to be present in previous research that has compared gamers to non-gamers [2,39]. In the present study, we took a novel approach to these issues by examining potential connections between a subset of cognitive skills (spatial working and long term memory) and MOBA gaming expertise by recruiting participants at an international MOBA gaming tournament. By doing so, we aimed to examine whether a wide range of gamer expertise can be captured at a gaming tournament, whether quality data can be collected in such an environment, and whether MOBA gaming expertise is connected to individual differences in cognitive skills.

Research examining the links between individual differences in cognitive skills and gaming expertise have yielded varying results. In these studies, participants are typically recruited from college campuses (majority of studies cited in this manuscript) and are separated into two groups of individuals based on gaming experience. Those who are defined as gamers typically play greater than five hours a week whereas non-gamers are defined as those who play less than an hour a week $[1,6,8,10,11,13,18,19,32]$. There is evidence that gamers outperform non-gamers in basic perceptual skills 
such as integrating information that is presented in multiple modalities [13], detecting changes in visual stimuli [10], and visuospatial acuity [19]. Differences have also been reported in visual attention capabilities with gamers being faster at finding a target amongst a set of distractors [8] and deploying attention to a select set of stimuli amongst distractors $[6,18]$. There is also mixed evidence that differences in gaming experience extend to visual working memory capacity defined as the number of chunks of visual information that can be retained [12]. Gamers outperformed non-gamers when given an N-back task where participants had to judge whether the current stimulus from a stream of randomly presented visual images was the same or different than the image N-back (e.g., two images back) [11]. Additional evidence of gaming experience effects has been observed with the speed of retrieving information in working memory [32]. However, there have also been null effects when examining the latent variables underlying working memory capacity [39]. A meta-analysis by Powers and colleagues [34] found that when there is an advantage for gamers over non-gamers on cognitive measures the effect size is typically small, which is consistent with mixed results in previous research.

There has been much discussion in regard to reconciling the mixed evidence of gamer advantages on cognitive tasks. The cross-sectional nature of the majority of gaming studies, placing participants into categories of gamers and nongamers, has been highlighted as a potential limitation for both statistical concerns [39] and threats to validity [2,24]. Specifically, setting up an extreme-group design which omits a large range of individuals between the two extreme categories can lead to increased risk of statistical errors including an increased chance of Type I error [35,39]. Additionally, since this research has typically grouped gamers into one category it is an open question as to whether similar relations exist with gamers of different expertise. To begin to address these issues we focused on individuals within the 'gamer' category. Previous research with chess experts has taken a similar approach, examining whether individuals who vary in chess experience also vary in performance on cognitive tasks. For example, it has been observed that chess players that range in skill, from beginners to chess masters, vary in their ability to chunk the spatial locations of chess pieces on a board $[9,16]$. By taking a similar approach, we aim to address the open question as to whether the reported effects in previous video game research that categorize participants as those who do and do not play games apply to gamers who range in expertise.

Addressing individual differences in gamer expertise requires a participant recruitment approach different from previous research. The majority of gaming studies have recruited participants from college campuses. This has led to small sample sizes of gamers in part due to the few participants who can be classified as gamers [39]. Furthermore, whereas individuals who play more than 5 hours per week are identified as gamers in these studies, expert eSports players can play that many hours in one day [29]. These issues cast doubt as to whether a sufficient sample size of gamers who range in expertise can be recruited at college campuses. We propose a novel approach: recruiting participants from international gaming tournaments. By targeting these events, we believe that a representative arc of skill levels within a game can be captured, providing a large and externally valid sample of gamers who vary in expertise.

International gaming tournaments that have been established with the rise of eSports offer an opportunity to recruit a large number of gamers that range in gaming expertise. Some of the most popular tournaments, in regard to attendance and viewership, are those of MOBA games such as League of Legends and Defense of the Ancients II (Dota 2). This genre of game is typically defined as being a free-to-play game where gamers control a hero they select and, alongside a team of other gamers and their heroes, battle an opponent team with the goal of destroying their home base. The tournaments are composed of brackets where teams compete against each other to win money and recognition. They are typically held in indoor arenas to fit both the teams competing and the large number of fans that attend to view the competition. Much like professional sporting events such as the NCAA College Basketball Championship, MOBA tournaments attract dedicated gamers who are interested in the game and vary in gaming expertise themselves. These fans are typically familiar with the basic mechanics of a game and are typically players of the game themselves. This offers the chance to sample gamers who are familiar with a particular game and vary from novices to professional gamers. However, recruiting participants in a tournament environment is much different than a laboratory environment. To our knowledge, running a cognitive research study at a gaming tournament has yet to be done although the value of it has been considered theoretically [23]. This leads to a basic but critical question: can valid and reliable data be collected at a MOBA tournament?

The MOBA genre is unique relative to the types of games that typically dominate gaming research (e.g., first person shooters, puzzle games such as Tetris) and emphasizes a different balance of skills [24]. Whereas FPS games emphasize precise coordination of visual and motor processes to detect and then react to opponents, MOBA players, to a larger extent, rely on in-depth knowledge of character capabilities to strategically select an appropriate match-long plan for defeating opponents. Furthermore, players adapt to the ever changing status of the battle arena. MOBA games contain a number of buildings and structures that can either be a danger or an opportunity to gain an advantage depending on game progression. As such, accurately and rapidly monitoring and responding to changes displayed via the arena map can give a competitive advantage. Given both the emphasis and importance of knowledge recall in MOBAs, they provide an opportunity to examine whether gaming expertise transfers beyond 
perceptual abilities and working memory skills, to long term memory. Specifically, the ability to recall which game elements are located where relies in part on spatial memory, a component of episodic memory [5]. This leads to the question as to whether MOBA gamers of higher expertise are better able to retrieve spatial memories compared to those gamers with less expertise.

The goal of the present study is to evaluate the use of a MOBA tournament environment to examine whether individual differences in gaming expertise are reflected in cognitive skills. To do so, we selected The International 5 (TI5) tournament of the game Dota 2 to examine whether gamers who vary in Dota 2 expertise also vary in working memory capacity and spatial memory performance. We tested working memory for two reasons. First, as reviewed above, there is mixed evidence as to whether gamers outperform non-gamers on measures of working memory (cf. $[11,39])$. As such, it is an open question as to whether this relation exists when looking within the MOBA gamer population. Second, there has been much research examining working memory performance in young adults $[22,33,38]$ providing a benchmark to which tournament participants can be compared to determine the reliability and validity of data collected at a gaming tournament. In regard to spatial memory, the emphasis of MOBAs on long term memory storage and recall make it an opportune genre to examine whether relations exist between spatial memory measures and gaming expertise. Patterns of performance on a pair of spatial memory tasks observed in previous research can be used to further assess the validity and reliability of the tournament data. Specifically, in these tasks participants are shown an array of pictures. Next they are presented a similar array of images where some of the spatial locations of the pictures have been changed. Participants have been found to be more accurate when recalling spatial locations when the objects have exchanged locations with each other compared to when objects are moved to novel locations where no object was previously located $[21,26]$. Examining whether a similar performance difference is present with tournament participants can provide an additional way to evaluate the quality of data collected in such an environment. To focus specifically on potential differences in working and spatial memory in relation to gaming expertise we also included a baseline measure of cognitive ability. We utilized a number ordering task that has been found in previous research to be related to math ability [28] to control for general cognitive performance of tournament participants.

An ancillary objective was to compare the use of objective versus self-report measures of gaming expertise. Gamers have typically been classified based on the amount of time spent playing a game. This raises the question as to whether self-report measures of play experience can be used as a proxy measure of expertise. Specifically, exposure does not necessarily equal expertise; a person who plays basketball frequently for exercise has much basketball experience, but may not be an expert basketball player $[2,24]$. Dota 2 is played online on a platform called Steam (Valve Corporation) which gathers a great deal of information about players and their game play. We were able to retrieve the Dota 2 player rankings that were generated via a proprietary algorithm Valve Corporation uses to rank player expertise similar to the Elo rating system for chess players. Additionally, we retrieved the amount of time gamers have played the game as recorded by the game itself. For selfreport measures of gamer expertise, we asked gamers to rate the length of their playing history with Dota and the amount of time they play the game per week. By including both gaming expertise measures we aimed to evaluate the extent to which these measures yield similar results to determine whether they can be independently used in future research to assess gaming experience.

We have the following hypotheses:

H1: Recruiting participants at a game tournament will result in a large, representative sample of gamers who vary in expertise.

$\mathrm{H} 2$ : Data collected within a gaming tournament environment will be of similar quality compared to cognitive tasks presented within laboratory environments.

H3: MOBA expertise will be correlated with individual differences in spatial working and long term memory.

H4: Objective and self-report measures of MOBA expertise will similarly relate to individual differences in working and spatial memory performance.

\section{METHOD}

\section{Study Environment}

The tournament took place inside Key Arena in Seattle, WA, USA from August 3rd to August 8th, 2015. Outside the arena was a large lawn area with a screen that had the tournament coverage projected onto it, a merchandise store, and a pavilion area that contained sponsors and vendors. It was within this vendor area that the data collection took place. The booth was situated adjacent to a video game demo area as well as a sponsored DJ. Booth space at TI5 for the study was provided by Valve Corporation. No tournament pass was required to enter the vendor area. It was open each day of the tournament from approximately 10:00am to 6:00pm.

\section{Hardware and Software}

All components of the study were presented via a set of tasks built using the Python-based OpenSesame experiment builder (v. 2.9.6) [31] and custom scripts. Laptops were used to run the study (screen diagonal of 13.3 in) and ran the Windows 8 operating system. Participants had to use both the laptop keyboard and an external wired mouse to complete the tasks. A total of five laptops were used to run up to five participants simultaneously.

\section{Participation Requirements}

To participate in the study, individuals had to meet the following requirements: they needed to speak and 
understand English, have knowledge of Dota 2, be able to provide their Steam account login ID, had to be attending or staffing the tournament (checked via TI5 badge), and either had to be 18 years of age or older or have signed parental permission to participate. For their participation, participants received either a t-shirt or collectible plush doll (donated by Valve Corporation).

\section{Demographics Questionnaire}

Near the end of the study, participants were requested to provide a set of demographic information including their age and years of schooling they had completed (as text-entry variables), ethnicity and race, gender, and to complete a series of questions used to indicate level of affluence. Affluence was assessed using the Family Affluence Scale for adolescents [4]. This 4-item scale asks participants a series of questions designed to measure the family wealth of individuals and responses to each question were summed together to create an overall affluence score with the following bands corresponding to different levels of wealth: low $=0-2$, middle $=3-5$, upper $=6-9$.

\section{Self-Reported Video Game Experience Measures}

Participants indicated their experience with video games via a series of questions. The length of their Dota play history (both Dota 1 and 2) was assessed using a 7-point Likert-type scale with the following labels corresponding a response of 0 to 7: "Never", "0-6 months", "6-12 months", "1-3 years", "3-6 years", "6-9 years", "9+ years". Since the study required participants to have played some Dota, individuals who responded "Never" were not included in analyses. Participants were also asked to indicate the number of hours per week they play video games and the percent of that time they play Dota 2 (both text-entry responses). Using these values, we calculated the number of hours participants typically played Dota per week.

\section{Objective Video Game Experience Measures}

To play Dota 2, individuals need to $\log$ into their Steam account and have an active internet connection. By doing so, match statistics are captured and saved to the player's account. Some of these measures are publicly accessible while others are only available to the player and Valve Corporation. We asked participants to provide their Steam ID to participate in the study and with the assistance of Valve Corporation we were able to collect objective measures of gamer experience and expertise. Specifically, we included total playtime which indicates the total amount of time a gamer has played Dota 2 (in hours). Additionally, we retrieved matchmaking rankings (MMR). This index of expertise is akin to the Elo rating system of chess players where the higher the ranking indicates a higher skill level player. The MMR rating we selected is generated by the results of ranked matches where players entered the match as an individual (as compared to part of a team). A player's MMR is used to match them against an opponent such that there is close to a $50 \%$ chance of either player winning a match.

\section{Cognitive Tasks}

Spatial Working Memory Task

The working memory task was designed to assess spatial working memory capacity and performance speed. It was based on the abbreviated symmetry operation span task developed by Oswald and colleagues [33] and the automated operation span tasks developed by Unsworth and colleagues [38]. For the task, participants had to recall the spatial positions of a set of red blocks that were randomly placed individually within a $4 \times 4$ grid [instructions: "For each trial, you will be presented with a set of three to five pairs of figures and red blocks. After the set, you will then be presented with a grid where you will have to recall where the red blocks were located and the order in which they were presented (from first to last presented block)."]. Before the presentation of each red block, participants had to make a symmetry judgment regarding a black and white block figure composed within an $8 \times 8$ grid. They had to judge whether the figure was symmetrical along the y-axis (approximately half of the figures were symmetrical). The symmetry judgment and red block pairs occurred from 3 to 5 times, after which participants were presented with a blank 4 x 4 grid and were instructed to click the boxes that contained the red blocks in the exact order in which they were presented (a total of 2 complete trials for 3, 4, and 5 sets; 24 individual block responses total).

The trial procedure was as follows: a fixation dot appeared for $800 \mathrm{~ms}$ after which the $8 \times 8$ symmetrical figure was presented. The duration of the response period depended on each participant's performance during the symmetry practice trials. Following Redick and colleagues [36], participants were presented with six practice trials performing the symmetry task where the symmetrical figure remained onscreen until they clicked one of two buttons to indicate whether it was or was not symmetrical. The mean reaction time was calculated for these trials and the duration for symmetry judgments on test trials was set as the mean plus $2.5^{*} S D$. Participants were informed of the time in which they were required to respond prior to the start of test trials. After the symmetry judgment, the $4 \times 4$ grid with one randomly placed red block was presented for $650 \mathrm{~ms}$. After which, the next symmetry judgment was presented if the number of pairs for the trial had yet to be reached, or, the response period was initiated. There was no time limit for the response period and immediately after the last response the next trial began.

This task utilized the shortened operation span task developed by Oswald and colleagues [33] ensuring that participants could complete the entire task in around 3 to 5 minutes. This task has been found to be valid and reliable (correlation with latent working memory capacity variable = .48; Cronbach's alpha $=.59$ [33]). Scoring for the working memory task followed the absolute scoring method as described by Conway and colleagues [12] which sums all blocks correctly recalled in position and order across trials. 
In addition, median reaction time for clicking each block in the recall portion of the task was collected. Overall accuracy on the symmetry task was used as a method for determining whether participants were following directions.

\section{Spatial Location Memory Task}

This task was utilized to assess facets of episodic memory, specifically spatial location memory. For this task participants were asked to memorize the locations of objects in two pictures (instructions: "For this task you will be presented with two pictures of sets of objects, one at a time. Your goal is to memorize the locations of all the objects in the two pictures so that you can detect which objects have moved locations."). Afterwards, participants were asked to identify which objects in each picture had moved locations or remained in the same location. The task was based on those used by James and Kimura [21] and Levy, Astur, and Frick [26]. Two experimental conditions were used. In the exchange condition, a subset of the objects (10 of the 19 objects) exchanged locations with another object in the picture. In the shift condition, a subset of the objects (10 of 19) moved to a novel, unoccupied location.

The procedure was as follows. After a $1000 \mathrm{~ms}$ fixation dot, participants were presented with the exchange picture for 30 seconds and were asked to memorize the locations of the objects. Immediately after, a fixation dot was presented for $1000 \mathrm{~ms}$ and the shift picture remained on screen for 30 seconds and participants were asked to memorize the locations of the objects. Each picture contained a unique set of objects. After the 30 seconds elapsed, a manipulated version of the exchange picture was presented on screen and participants were asked to indicate for each object whether it had shifted locations or remained in the same place using a computer mouse. Participants left-clicked to indicate the object remained in the same location (a blue rectangle marked the object when left-clicked) and right-clicked to indicate the object had moved locations (a red circle marked the object when right-clicked). Participants had an unlimited amount of time to click all objects on the screen but were asked to work quickly and efficiently. They could also change the marking on each object as many times as they wanted. Once they had marked all objects, they could proceed by clicking a "continue" button. Next, a fixation dot appeared for $1000 \mathrm{~ms}$ and participants performed the same set of judgments for the shift condition picture. The dependent variables generated for this task were the sensitivity measure d-prime for each picture condition [30] and response time (RT) of the recall period (from onset of picture to clicking the 'continue' button) for each condition. The d-prime measure incorporates the hit rate (correctly saying a target had changed locations) and false alarm rate (incorrectly saying a target had changed locations) to assess how sensitive a participant was to a change in object location while controlling for guessing.

\section{Number Order Task}

This task was used to assess participants' numerical processing ability which served as a baseline measure of cognitive processing. This was done for two reasons. First, we wanted our baseline measure to require participants to evaluate the semantic content of stimuli rather than a simple stimulus-response decision. Second, performance on symbolic number tasks has been found to be significantly related to a set of cognitive measures including arithmetic and working memory making it a valid measure of cognitive performance [27,37]. For this task, modeling the ordinal task utilized by Lyons and Beilock [28], participants viewed three Arabic numerals that ranged from 1 to 9 arranged horizontally and had to judge whether or not they were increasing in numerical value from left to right (instructions: "In this part of the experiment you will be presented with three numbers each from 1 to 9 . Your task is to judge whether the numbers are increasing in value from left-to-right."). A total of 36 test trials were presented, half of which contained numerals that were increasing from left to right and half that were not (half of these trials were randomly arranged while the other half decreased from left to right). Participants were required to respond within 1.5 seconds of viewing the numerals (pressed the ' $\mathrm{O}$ ' key if numbers increased from left to right, ' $\mathrm{P}$ ' key if they did not). If no response was made, the trial was marked as incorrect. Overall accuracy of correctly responding increasing or decreasing orders and median reaction time (RT) for correct trials were used as dependent variables for this task.

\section{Study Procedure}

To participate in the study, participants were first told what the study entailed and, if they remained interested, they filled out a release form. If a laptop was available, the participant was seated. If not, they were told to come back at a certain time to participate. Once seated at the laptop, participants were asked to read the instructions for each task carefully and to ask questions if they had any. They were then offered ear plugs to use if they thought it would help them concentrate on the tasks in the midst of the noise generated by the vendors and attendees of the tournament. Participants were then presented with an electronic consent form that abided by the guidelines of the Declaration of Helsinki. They then completed a set of cognitive tasks, in a fixed order, containing the spatial working memory task, location memory task, numerical ordering task, and demographic information. After they completed these tasks they were presented with an electronic debriefing. Finally, participants were presented with an incentive (t-shirt or plush doll). The entire study took approximately 20 to 30 minutes to complete. 


\begin{tabular}{ccc}
\hline Race & $\begin{array}{c}\text { Hispanic or } \\
\text { Latino }\end{array}$ & $\begin{array}{c}\text { Not Hispanic or } \\
\text { Latino }\end{array}$ \\
\hline American Indian & 3 & 3 \\
Asian & 0 & 130 \\
Black & 0 & 1 \\
Pacific Islander & 0 & 1 \\
White & 16 & 198 \\
Multiracial & 5 & 28 \\
\hline
\end{tabular}

Table 1. Frequency of participants in race by ethnicity categories.

\section{RESULTS}

\section{Participant Characteristics}

A total of 396 participants provided complete data for each of the cognitive tasks. A total of 34 females, 360 males, and 2 individuals who identified as "other" were recruited. The average age of participants was 23.41 years old $(S D=4.12$, Min = 13, Max = 50). Of the participants, 368 provided valid education level information with 28 completing at least some graduate schooling, 257 completing at least some college, 70 completing at least some high school, and 13 completing at least some middle school. Participants who provided racial and ethnic information were from a range of backgrounds (see Table 1). The majority of participants $(69.95 \%)$ fell within the upper band of the affluence scale $(M=6.18, S D=$ 1.53). All subsequent statistical tests were two-tailed with $\alpha$ $=.05$.

\section{Evaluating Quality of Data Collected at Tournament}

We examined the quality of the data collected at the gaming tournament using two sets of analyses. First, we evaluated the performance rates of each task and the rate of attrition due to outliers. Second we analyzed the reliability of each task and, if available, compared these results to previous research

\section{Working Memory Task}

Six participants did not maintain the $85 \%$ accuracy cutoff for the symmetry judgments as recommended in previous research [38]. An additional 14 participants were identified as outliers by being beyond $3 * S D$ from the mean for either block score $(M=19.26, S D=3.89)$ or median reaction time for block recall $(M=1783.27 \mathrm{~ms}, S D=415.13$; retained $N=$ 376 ; attrition rate $=5.1 \%$ ).

We next compared the scores in the present study to those collected by Oswald and colleagues [33] from a college campus. Participants performed significantly higher in the present study with a $95 \%$ confidence interval of 18.86 to 19.65 compared to an estimated interval of 15.26 to 16.56 in Oswald et al. (2015). Despite this difference, the observed reliability of block score in the present study $($ alpha $=0.61)$ was comparable to that observed in Oswald et al. (2015), alpha $=0.59$.
Location Memory Task

Thirteen participants were identified as outliers by being outside $3 * S D$ from the mean for overall accuracy and response time for either picture condition (retained $N=383$; attrition rate $=3.3 \%$ ). Reliability based on accuracy for each item in both conditions was acceptable (alpha $=0.65)$.

In line with previous research [21,26] participants were marginally more sensitive to changes in the exchange condition with higher d-prime scores $(M=0.77, S D=0.80)$ compared to the shift condition $(M=0.68, S D=0.76), t(382)$ $=1.84, p=0.067$. Additionally, response times were significantly shorter in the shift $(M=26881.04 \mathrm{~ms}, S D=$ 9724.36) versus exchange $(M=39662.57 \mathrm{~ms}, S D=$ 13062.70) condition, $t(382)=-20.76, p<0.001$.

\section{Number Order Task}

Two participants were identified as either not paying attention to directions by having less than $50 \%$ accuracy or outliers by being outside $3^{*} S D$ from the mean for median reaction time (retained $N=394$; attrition rate $=0.1 \%$; accuracy: $M=0.86, S D=0.10$ ); RT: $M=775.45 \mathrm{~ms}, S D=$ 118.87). Reliability based on accuracy for each trial in the task was acceptable $($ alpha $=0.71)$. Additionally, accuracy was significantly above chance, $t(393)=74.63, p<.001$.

\section{Summary}

When analyzing performance on the cognitive tasks we observed low attrition rates and acceptable reliability. Additionally, performance was above chance for the number order task indicating participants were following task instructions.

\section{Initial Comparisons of Performance Across Gaming Measures and Cognitive Tasks}

Prior to conducting correlational analyses, participants with missing or invalid data or who were identified as outliers in the tasks above were excluded from further analyses (retained $N=362$ ). A total of 116 participants were dropped for either not having provided a valid Steam ID (required to retrieve MMR) or for not having played a ranked match. One participant was identified as having a total playtime more than twice the duration of the next highest participant and was removed from further analyses. Upon inspecting the remaining distribution of scores four measures were identified as having extreme skew (age, skew $=1.04$; total playtime, skew $=1.64$; block RT, skew $=1.19$; Dota per week, skew $=1.89$ ) and were transformed to improve the normality of the distribution [age transformed using age $=$ sqrt (age), block RT transformed using blockRT $=\log ($ blockRT), total playtime transformed using totalplaytime $=$ sqrt(totalplaytime), Dota per week transformed using Dotaperweek=sqrt(Dotaperweek); see Table 2 for percentiles for game measures]. 


\begin{tabular}{cccccc}
\hline & $\mathbf{5 \%}$ & $\mathbf{2 5 \%}$ & $\mathbf{5 0 \%}$ & $\mathbf{7 5 \%}$ & $\mathbf{9 5 \%}$ \\
\hline MMR & 2254 & 3000 & 3526 & 4019 & 4710 \\
$\begin{array}{c}\text { Total Playtime } \\
\text { (hours) }\end{array}$ & 625 & 1518 & 2324 & 3387 & 6082 \\
$\begin{array}{c}\text { Dota per week } \\
\text { (hours) }\end{array}$ & 3 & 8 & 14 & 23 & 45 \\
\hline
\end{tabular}

Table 2. Percentile scores for gaming measures (no transformation applied; $\mathrm{MMR}=$ match making ranking).

Zero-order Correlations across Gaming Experience and Cognitive Task Measures

We initially examined whether individual differences in measures of gaming experience correlated with performance on the cognitive tasks. We observed that MMR was significantly positively correlated with age and number task accuracy, Dota history length was significantly positively correlated with age, and Dota per week was significantly negatively correlated with block score, age, and number task accuracy (see Table 3).

\section{Approach for Analyzing Contribution of Dota 2} Experience to Cognitive Task Performance

To assess the extent to which cognitive performance was predicted by gaming experience we used a set of step-wise multiple regression models. In all models the first step included age, number task RT, and number task accuracy to account for developmental and general cognitive factors when predicting task performance. The second step differed according to whether objective measures of Dota 2 experience (MMR and total playtime) or self-report measures (Dota per week and Dota history length) were used. Using this analysis, the contributions of Dota 2 measures were analyzed for working memory and location memory task performance. All beta coefficients presented are standardized and are summarized in Table 4.
Predicting task performance with objective measures of gaming experience.

Of the models predicting cognitive task measures, MMR was a significant predictor of memory shift RT, $\beta=-0.166, t(230)$ $=-2.228, p=0.027$. Total playtime was not a significant predictor of any measure.

Predicting task performance with subjective measures of gaming experience.

When predicting cognitive task measures, Dota per week was a marginal predictor of block score, $\beta=-0.113, t(230)=$ $-1.7, p=0.09$. Dota history length was a marginal predictor of memory shift RT, $\beta=-0.119, t(230)=-1.703, p=0.09$.

\section{DISCUSSION}

The goal of the present study was to evaluate the use of participants at a gaming tournament to examine whether MOBA gaming expertise is related to cognitive skills. Over the span of six days we collected data from close to 400 gamers as they completed working memory, spatial memory, and number ordering tasks. Using reliability statistics and previous research as a benchmark for evaluating the data collected during the tournament, we found evidence of valid and reliable data for a set of cognitive tasks. In addition, we examined the use of self-reported and game-generated measures of gamer expertise in relation to performance on these tasks. We observed a similar finding for both sets of gaming measures for the location memory task: participants who had higher MMR or a higher amount of Dota 2 played per week had faster response times on the shift condition task. However, the two sets of measures deviated for the working memory task with self-reported Dota history length being a marginal predictor of block score. We argue that these results indicate that recruiting gamers at a tournament can provide a participant sample with a varied range of gaming expertise, and that MOBA gaming expertise is weakly correlated with individual differences in the speed of spatial long term memory recall.

\begin{tabular}{|c|c|c|c|c|c|c|c|c|c|c|c|c|c|}
\hline & $\begin{array}{l}1 \\
\text { MMR }\end{array}$ & $\begin{array}{l}2 \\
\text { Total } \\
\text { Playtime }\end{array}$ & $\begin{array}{l}3 \\
\text { Dota } \\
\text { History } \\
\text { Length }\end{array}$ & $\begin{array}{l}4 \\
\text { Dota per } \\
\text { week }\end{array}$ & $\begin{array}{l}5 \\
\text { Block } \\
\text { Score }\end{array}$ & $\begin{array}{l}6 \\
\text { Block } \\
\text { RT }\end{array}$ & $\begin{array}{l}7 \\
\text { Shift d- } \\
\text { prime }\end{array}$ & $\begin{array}{l}8 \\
\text { Shift RT }\end{array}$ & $\begin{array}{l}9 \\
\text { Exch. d- } \\
\text { prime }\end{array}$ & $\begin{array}{l}10 \\
\text { Exch. } \\
\text { RT }\end{array}$ & $\begin{array}{l}11 \\
\text { Age }\end{array}$ & $\begin{array}{l}12 \\
\text { Number } \\
\text { Task } \\
\text { Acc. }\end{array}$ & $\begin{array}{l}13 \\
\text { Number } \\
\text { Task } \\
\text { RT }\end{array}$ \\
\hline 2 & $.409^{* * *}$ & - & - & - & - & - & - & - & - & - & - & - & - \\
\hline 3 & $.367^{* * *}$ & $.143^{*}$ & - & - & - & - & - & - & - & - & - & - & - \\
\hline 4 & .047 & $.370^{* * *}$ & -.079 & - & - & - & - & - & - & - & - & - & - \\
\hline 5 & .028 & -.016 & -.016 & $-.139^{*}$ & - & - & - & - & - & - & - & - & - \\
\hline 6 & -.036 & .080 & .124 & .031 & $-.347^{* * *}$ & - & - & - & - & - & - & - & - \\
\hline 7 & -.056 & -.080 & -.026 & .037 & $.143^{*}$ & -.071 & - & - & - & - & - & - & - \\
\hline 8 & -.100 & .052 & -.076 & -.024 & .040 & $.311^{* * *}$ & $.224^{* * *}$ & - & - & - & - & - & - \\
\hline 9 & -.040 & -.103 & -.058 & .053 & .046 & .030 & $.379^{* * *}$ & $.262^{* * *}$ & - & - & - & - & - \\
\hline 10 & -.097 & .006 & .104 & -.062 & -.074 & $.262^{* * *}$ & .077 & $.453^{* * *}$ & .107 & - & - & - & - \\
\hline 11 & $.184^{* *}$ & .098 & $.386^{* * *}$ & $-.195^{* *}$ & -.035 & .109 & -.003 & .063 & .039 & .103 & - & - & - \\
\hline 12 & $.242^{* * *}$ & -.039 & .062 & $-.203^{* *}$ & $.210^{* *}$ & -.110 & .087 & .045 & .111 & -.066 & $.202^{* *}$ & - & - \\
\hline 13 & -.105 & .089 & .019 & .095 & -.109 & $.278^{* * *}$ & .005 & $.144^{*}$ & .127 & $.172^{* *}$ & .023 & $-.201^{* *}$ & - \\
\hline
\end{tabular}

Table 3. Correlations between objective (MMR= match making ranking; Total Playtime) and subjective (Dota History Length; Dota per week) gamer expertise measures and cognitive task performance $($ Exch $=$ Exchange $)$. 


\begin{tabular}{|c|c|c|c|c|c|c|c|}
\hline & & $\begin{array}{l}\text { Block } \\
\text { Score }\end{array}$ & Block RT & $\begin{array}{l}\text { Shift d- } \\
\text { prime }\end{array}$ & $\begin{array}{l}\text { Exch. d- } \\
\text { prime }\end{array}$ & Shift RT & Exch. RT \\
\hline \multirow{3}{*}{$\begin{array}{c}\text { Step 1 } \\
\text { Predictors }\end{array}$} & Age & -.076 & $.119^{+}$ & -.023 & .007 & .046 & $.111+$ \\
\hline & Num. Task Acc. & $.212^{* *}$ & -.082 & .097 & $.140^{*}$ & .067 & -.057 \\
\hline & Num. Task RT & -.064 & $.259^{* * *}$ & .025 & $.155^{*}$ & $.156^{*}$ & $.158^{*}$ \\
\hline \multicolumn{8}{|c|}{ Model with Objective Dota Expertise Measures } \\
\hline Step 2 & MMR & -.025 & -.037 & -.055 & -.017 & $-.166^{*}$ & -.104 \\
\hline Predictors & Total Playtime & .016 & .058 & -.055 & -.107 & .106 & .022 \\
\hline \multicolumn{8}{|c|}{ Model with Subjective Dota Expertise Measures } \\
\hline \multirow{2}{*}{$\begin{array}{c}\text { Step } 2 \\
\text { Predictors }\end{array}$} & Dota per Week & $-.113^{+}$ & .015 & .053 & .073 & -.018 & -.072 \\
\hline & $\begin{array}{l}\text { Dota History } \\
\text { Length }\end{array}$ & .001 & .092 & -.027 & -.085 & $-.119^{+}$ & .072 \\
\hline
\end{tabular}

Table 4. Standardized beta coefficients for step-wise regression predictors (MMR = match making ranking; Exch = Exchange; Num $=$ Number $)$.

Game Expertise of Participants Recruited at Tournament Compared to College Campuses

The results of the present study suggest that gaming tournaments provide an opportunity to collect valid data from a large number and range of gaming experts. A motivating factor for the present study was to examine whether a representative spread of gamer skill levels, from novice through intermediate up to expert, could be recruited from a gaming tournament. When comparing the sample of gamers that we recruited from the tournament to previous research, two main differences are observed. First, the amount of video game play time was much higher for the tournament participants who were used in the regression analyses. Participants at the 25 th percentile reported playing 8 hours per week, which is more than the typical lower boundary for defining gamers at college campuses ( 6 hours; see Introduction), and the 75th percentile playing 23 hours per week. This suggests that the sample of gamers recruited at the tournament had a higher level and broader range of gaming expertise compared to samples recruited in previous research $[1,6,8,10,11,13,18,19,32]$. In addition, we were able to utilize the in-game metrics to confirm an array of skill levels from MMR scores, ranging from 1088 to 6006 (see

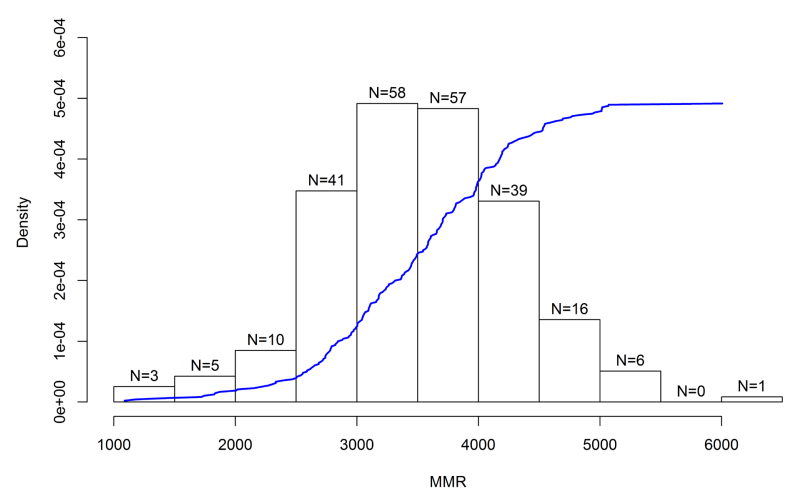

Figure 1. Cumulative distribution function (blue) and histogram ( $N$ = number of participants within bin) for match making ranking (MMR).
Figure 1). This suggests that recruiting participants from a gaming tournament provided a range of gamers that differ in experience and expertise. Although this is not surprising given data collection occurred at a gaming tournament, this can provide justification for future research to use eSport events as a method to recruit skilled gamers.

\section{Evidence That Quality Data Can Be Collected at Game Tournaments}

The results of the working memory and location memory tasks suggest that the data collected from tournament participants were valid and reliable. For the working memory task, the calculated reliability coefficient was close to what has been reported in previous research using the shortened symmetry span task with participants recruited from college campuses [33]. Furthermore, participants' block score performance further indicates the data was valid. One prediction is that if participants were providing low quality data their scores would be lower than those reported in previous research due to a number of potential factors (e.g., low motivation, distractions). However, the $95 \%$ confidence interval for working memory performance was higher than reported scores collected at college campuses [33]. It is also notable that the attrition rate for failing to maintain the $85 \%$ accuracy threshold for the symmetry judgments was quite low $(2 \%, 6$ out of 396 participants) and the attrition rate remained acceptable when factoring in an additional 14 participants who were outliers for median reaction time and block score $(5 \%, 20$ out of 396 participants). Although, to our knowledge, the attrition rates for college students completing the shortened version of the automated operation span tasks have not been reported, the attrition rate is lower than what has been reported for other versions of the task (e.g., [38]). Results from the location memory task also provide evidence that tournament participants provided valid data. Estimated reliability was adequate, attrition rates were low, and there were marginally higher d-prime scores on the exchange versus the shift condition. Although the effect was marginal, it is in the same direction as has been reported in previous research $[21,26]$. A concern, however, was the fixed sequence of the shift condition coming after the exchange 
condition could account for the performance difference. Additionally, performance was relatively low for each condition as indicated by low d-prime scores. However, results from the number task provide additional evidence of valid data. Again, estimated reliability was adequate and attrition rates were low. Furthermore, there was a significant positive correlation between number task accuracy and working memory block score which is in line with previous research [27]. Overall, we believe that these results indicate that the data collected was valid and reliable.

We would like to emphasize that recruiting participants from a gaming tournament should be viewed as a complementary approach to recruiting participants from a college campus and conducting research in a controlled laboratory environment. Although the sample of gaming experts has higher external validity when recruiting from a gaming tournament in regard to obtaining a range of gamer expertise, a laboratory setting is most likely better suited to run gaming expertise studies that focus on more perceptual skills (e.g., attentional blink). As such it is important to consider the requirements and goal of the study when deciding whether it is more advantageous to recruit and test participants at a gaming tournament or college campus.

\section{Challenges and Recommendations When Recruiting Participants at Gaming Tournaments}

When running this study at a gaming tournament we encountered two main challenges when running participants. First, environmental noise and distractions were inherent in the tournament environment. The noise was primarily generated from attendees and other vendors in the outside pavilion area and the distractions also included environmental factors such as glare on computer screens from the sun. To mitigate these, we gave participants the option of using disposable earplugs and cardboard partitions to shade computers from the sun. An additional challenge we encountered was the sheer number of attendees that were interested in participating. Although testing a large number of participants in a short time period was a strength of this approach, the logistical challenge was determining how to assign participants a time-slot for testing. Our approach, which can be improved upon, was if the testing computers were occupied to have interested participants sign-up for a timeslot later in the day. We recommend a similar approach for future research.

Given the range of different eSport tournaments we believe that researchers will be able to identify events that are best suited for their research goals and available resources. Tournaments occur at different geographical scales (e.g., local, regional, international) as well as with different genres (e.g., MOBAs, first person shooters; [17]) making them a rich opportunity to ask a research question with a specific gamer population in mind. Additionally, while ideally tournament organizers would be supportive, there are ways to conduct research alongside a tournament. For example, an alternative approach would have been to locate the testing area in a location either adjacent to the tournament venue or at tournament affiliated locations (e.g., hotels). Combined with utilizing social media or handing out flyers we believe this would be an effective way to conduct research without being onsite at a tournament.

A concern with the data we collected is the high level of missing scores in regard to self-reported and objective measures of gaming experience. This occurred primarily for two reasons. First, participants were requested to provide the number of hours per week that they played video games and the percent of time they played Dota 2, each using a text response. Some of the entries were invalid or unclear leading to missing Dota 2 per week measures. Second, we requested that participants write down their Steam user account ID that we later used to retrieve objective game statistics. Some of the IDs provided were invalid (e.g., misspelled, wrong account name) and further some of the participants had not played a ranked game which is required to generate a ranked MMR. We believe that this should not discourage future studies from utilizing such measures since relatively minor adjustments can be made to reduce the amount of missing data. For self-reported measures of gaming experience, using a forced-selection method (e.g., drop-down box with percentages) could reduce missing data. Furthermore, a system for obtaining objective measures, including private measures such as MMR, of gaming experience would be to have participants log into their gaming accounts onsite to record these measures prior to the start of the study.

\section{Individual Differences in MOBA Expertise and Cognitive Skills}

A subset of cognitive task measures was weakly correlated with specific MOBA expertise measures. We observed that those with greater MOBA expertise, as indicated by MMR and Dota history length, were more likely to have faster response times on the memory shift condition. That the link was specific to the shift compared to the exchange condition suggests that this connection may be specific to spatial longterm memory. Whereas it has been argued that performance on the exchange condition relies more heavily on processes involved in object recognition, the shift condition has been argued to more heavily rely on spatial long-term memory $[21,26]$. Similar to tracking the locations of players in MOBAs, objects in the shift condition are moving to novel locations requiring participants not only have to determine whether the identity of objects has shifted (e.g., in MOBA, has this immovable structure been destroyed), but also if the location of the object was novel (e.g., in MOBA, have players moved to a new location). This result extends previous research that has reported a correlation between gaming expertise and the speed of processing [32] to spatial memory. It is important to note that in the regression analyses age as well as general processing ability (number task performance) were included as predictors. Controlling for these factors makes it unlikely that the relation between MOBA expertise and shift location RT were due to systematic variations in these variables. The size of the 
effects for MOBA experience and expertise measures were weak which is in line with the small to medium effect sizes reported in a meta-analysis of gamer versus non-gamer differences in cognitive skills [34]. To our knowledge the present study offers unique evidence that MOBA gaming expertise may be connected, in part, to spatial memory.

There was evidence of a trend for individuals who reported playing more Dota 2 per week being more likely to have lower block scores on the working memory task. Although this effect was marginal, the negative relation deviates from previous research that has found a positive relation between working memory task performance and gaming experience $[11,32]$ and research that has found no significant relation [39]. We are cautious in interpreting this result given the marginal nature of the effect. It is possible the high level of gaming experience we observed in our study could be a contributing factor. As mentioned previously, the number of hours played by participants (95th percentile was 45 hours per week) was much higher than rates reported in previous research. With age and other measures of cognitive performance controlled for in regressions, this pattern could suggest that there is a point of diminishing return in regard to cognitive skills with high levels of video game playtime. It has been found that excessive gaming playtime can be connected to lower scores on cognitive tasks and school performance $[14,15]$. Future research should attempt to replicate this effect and, if so, consider whether previously observed relations between cognitive skills and gaming experience differ for gamers at the lower versus higher end of the experience spectrum.

These results do not provide evidence regarding the causal direction of the relation between cognitive skills and gaming expertise. As highlighted by Boot and colleagues [3], certain cognitive traits could predispose individuals to select to play video games. This seems especially likely with MOBAs. The games are typically free-to-play and available to any individual who has a computer with internet access. Combined with a commonly perceived steep learning curve, due to factors like more limited in-game resources, additional gameplay mechanics like "denying", and additional character growth options compared to other MOBAs, it seems likely that there is a cognitive and motivational barrier to extended play of Dota 2. There is an array of individual characteristics, including cognitive and other traits (e.g., motivation; [42]), that make it more likely a player will invest the time and energy required to develop expertise within a game. It seems likely that the links observed in the present study are the result of gaming experts having the initial cognitive skill set and motivation to initially play the game well enough to continue and the resultant effects of accumulated experience with the game.

A final question is whether self-report and objective measures of MOBA experience were capturing gaming expertise to a similar degree. Interestingly, the strongest correlations between gaming measures were between self- reported Dota history length and MMR whereas Dota per week paired with total hours playing Dota 2. This suggests that the overall pattern of self-reported measures is similar to objective measures of gaming experience. Overall, these results indicate that specific measures of MOBA expertise (e.g., MMR) can be partially captured by collecting selfreported measures of MOBA history experience. However, researchers should consider whether reducing redundancy by including fewer gaming experience measures outweighs the additional variance potentially explained by including a range of measures.

\section{Future Directions}

Our study brought up several findings we believe will be useful both in practical applications and future research. First, our findings indicate that the growing industry of eSports is an opportunity for researchers to reach a different segment of gamers, particularly "hardcore" gamers. It is likely that this trend can extend beyond just gaming tournaments into the larger segment of gaming events in general (e.g., Electronic Entertainment Expo, Penny Arcade Expo). Using these venues, future research can address three questions that stand out from the present study. Specifically, the extent to which spatial memory is linked to gamer expertise relative to knowledge of game mechanics (e.g., semantic memory) is unclear. It may be that, when accounting for this semantic memory, spatial memory may have little if any correlation. Second, it is unclear which stage of memory recall (encoding, storage, consolidation, retrieval) contributed to the correlation. A possibility is that avid gamers may have an advantage during encoding by being better able to focus attention on relevant information despite noise from the environment. Finally, the potential for gender differences in the observed correlations should be considered. This will likely require a specific participant recruitment strategy that emphasizes equal sample sizes from each gender given that an open recruitment strategy which was used in the present study yielded a sample where the vast majority of the participants were male.

\section{CONCLUSION}

The present study provides evidence that gaming expertise studies can be conducted at MOBA gaming tournaments. Furthermore, we find evidence that individuals with higher levels of MOBA gaming expertise respond faster to decisions that rely on spatial memory. We suggest that researchers should consider conducting research at gaming tournaments in addition to college campuses to gain greater access to gaming experts.

\section{ACKNOWLEDGMENTS}

We would like to thank foundry10 and Valve Corporation staff for their assistance. Foundry10 is funded by Gabe Newell who also acts as the CEO of Valve Corporation, maker of Dota 2.

\section{REFERENCES}

1. L. Gregory Appelbaum, Matthew S. Cain, Elise F. Darling, Stephen R. Mitroff. 2013. Action video game playing is associated with improved visual sensitivity, 
but not alterations in visual sensory memory. Atten Percept Psychophys 75, 6: 1161-1167.

http://dx.doi.org/10.3758/s13414-013-0472-7

2. Walter R. Boot, Daniel P. Blakely, Daniel J. Simons. 2011. Do action video games improve perception and cognition? Frontiers in Psychology. http://dx.doi.org/10.3389/fpsyg.2011.00226

3. Walter R. Boot, Arthur F. Kramer, Daniel J. Simons, Monica Fabiani, Gabriele Gratton. 2008. The effects of video game playing on attention, memory, and executive control. Acta Psychologica 129: 387-398. http://dx.doi.org/10.1016/j.actpsy.2008.09.005

4. William Boyce, Torbjorn Torsheim, Candace Currie, Alessio Zambon. 2006. The Family Affluence Scale as a measure of national wealth: Validation of an adolescent self-report measure. Social Indicators Research 78: 473487. http://dx.doi.org/10.1007/s11205-005-1607-6

5. Neil Burgess, Eleanor A. Maguire, John O'Keefe. 2002. The human hippocampus and spatial and episodic memory. Neuron 35: 625-641. http://dx.doi.org/10.1016/S0896-6273(02)00830-9

6. Matthew S. Cain, William Prinzmetal, Arthur P. Shimamura, Ayelet N. Landau. (2014). Improved control of exogenous attention in action video game players. Frontiers in Psychology. http://dx.doi.org/10.3389/fpsyg.2014.00069

7. Ben Casselman. 2015. Resistance is futile: eSports is massive ... and growing. Retrieved September 23, 2015 from

http://espn.go.com/espn/story//id/13059210/esportsmassive-industry-growing

8. Alan D. Castel, Jay Pratt, Emily Drummond. 2005. The effects of action video game experience on the time course of inhibition of return and the efficiency of visual search. Acta Psychologica 199: 217-230.

http://dx.doi.org/10.1016/j.actpsy.2005.02.004

9. William G. Chase, Herbert A. Simon. 1973. Perception in chess. Cognitive Psychology 4: 55-81. http://dx.doi.org/10.1016/0010-0285(73)90004-2

10. Kait Clark, Mathias S. Fleck, Stephen R. Mitroff. 2011. Enhanced change detection performance reveals improved strategy use in avid action video game players. Acta Psychologica 136: 67-72.

http://dx.doi.org/10.1016/j.actpsy.2010.10.003

11. Lorenza S. Colzato, Wery P. M. van den Wildenberg, Sharon Zmigrod, Bernhard Hommel. 2013. Action video gaming and cognitive control: Playing first person shooter games is associated with improvement in working memory but not action inhibition. Psychological Research 77: 234-239. http://dx.doi.org/10.1007/s00426-012-0415-2

12. Andrew R. A. Conway, Michael J. Kane, Michael F. Bunting, D. Zach Hambrick, Oliver Wilhelm, Randall
W. Engle. 2005. Working memory span tasks: A methodological review and user's guide. Psychonomic Bulletin \& Review 12, 5: 769-786.

http://dx.doi.org/10.3758/BF03196772

13. Sarah E. Donohue, Marty G. Woldorff, Stephen R. Mitroff. 2010. Video game players show more precise multisensory temporal processing abilities. Attention, Perception, \& Psychophysics 72, 4: 1120-1129. http://dx.doi.org/10.3758/APP.72.4.1120

14. Douglas A. Gentile. 2009. Pathological video-game use among youth ages 8 to 18: A national study. Psychological Science 20, 5: 594-602. http://dx.doi.org/10.1111/j.1467-9280.2009.02340.x

15. Douglas A. Gentile, Hyekyung Choo, Albert Liau, Timothy Sim, Dongdong Li, Daniel Fung, Angeline Khoo. 2011. Pathological video game use among youths: A two-year longitudinal study. Pediatrics 127 , 2: E319-e329. http://dx.doi.org/10.1542/peds.2010-1353

16. Fernand Gobet, Herbert A. Simon. 1996. Expert Chess memory: Revisiting the chunking hypothesis. Memory 6: 225-255. http://dx.doi.org/10.1080/741942359

17. GosuGamers. 2015. eSports Gaming Events. Retrieved December 17, 2015 from

http://www.gosugamers.net/events

18. C.S. Green, Daphne Bavelier. 2003. Action video game modifies visual selective attention. Nature 423, 29: 534537.

19. C.S. Green and D. Bavelier. 2007. Action-video-game experience alters the spatial resolution of vision. Psychol Sci 18, 1: 88-94. http://dx.doi.org/10.1111/j.14679280.2007.01853.x

20. Leah Jackson. 2013. The rise of esports in America. Retrieved September 23, 2015 from http://www.ign.com/articles/2013/07/25/the-rise-ofesports-in-america

21. Thomas W. James, Doreen Kimura. 1997. Sex differences in remembering the locations of objects in an array: Location-shifts versus location-exchanges. Evolution and Human Behavior 18, 3: 155-163. http://dx.doi.org/10.1016/S1090-5138(97)00004-4

22. Michael J. Kane, David Z. Hambrick, Stephen W. Tuholski, Oliver Wilhelm, Tabitha W. Payne, Randall W. Engle. 2004. The Generality of working memory capacity: A latent-variable approach to verbal and visuospatial memory span and reasoning. J Exp Psychol Gen 133, 2: 189-217. http://dx.doi.org/10.1037/00963445.133.2.189

23. Petri Lankoski, Staffan Björk. 2015. Game Research Methods. ETC Press.

24. Andrew J. Latham, Lucy L. M. Patston, Lynette J. Tippett. 2013. Just how expert are "expert" video-game players? Assessing the experience and expertise of 
video-game players across "action" video-game genres. Frontiers in Psychology.

http://dx.doi.org/10.3389/fpsyg.2013.00941

25. Amanda Lenhart, Sydney Jones, Alexandra Macgill. 2008. Adults and video games. Pew Internet \& American Life Project. Retrieved September 23, 2015 from http://www.pewinternet.org/2008/12/07/adultsand-video-games/

26. Lauren J. Levy, Robert S. Astur, Karyn M. Frick. 2005. Men and women differ in object memory but not performance of a virtual radial maze. Behavioral Neuroscience 119, 4: 853-862.

http://dx.doi.org/10.1037/0735-7044.119.4.853

27. Ian M. Lyons, Sian L. Beilock. 2009. Beyond quantity: Individual differences in working memory and the ordinal understanding of numerical symbols. Cognition 113, 2: 189-204.

http://dx.doi.org/10.1016/j.cognition.2009.08.003

28. Ian M. Lyons, Sian L. Beilock. 2011. Numerical ordering ability mediates the relation between numbersense and arithmetic competence. Cognition 121, 2: 256261. http://dx.doi.org/10.1016/j.cognition.2011.07.009

29. Patrick Mackey. 2013. The summoner's guidebook: How to be the best at League of Legends. Retrieved September 23, 2015 from http://www.engadget.com/2013/08/15/the-summonersguidebook-how-to-be-the-best-at-league-of-legend/

30. Neil A. Macmillan, C. Douglas Creelman. 2004. Detection theory: A user's guide. Psychology Press.

31. Sebastiaan Mathôt, Daniel Schreij, Jan Theeuwes. 2012. OpenSesame: An open-source, graphical experiment builder for the social sciences. Behavior Research Methods 44, 2: 314-324.

http://dx.doi.org/10.3758/s13428-011-0168-7

32. Ashley F. McDermott, Daphne Bavelier, C.S. Green. 2014. Memory abilities in action video game players Computers in Human Behavior 34: 69-78. http://dx.doi.org/10.1016/j.chb.2014.01.018

33. Frederick L. Oswald, Samuel T. McAbee, Thomas S. Redick, David Z. Hambrick. 2014. The development of a short domain-general measure of working memory capacity. Behav Res. http://dx.doi.org/10.3758/s13428014-0543-2

34. Kasey L. Powers, Patricia J. Brooks, Naomi J. Aldrich, Melissa A. Palladino, Louis Alfieri. 2013. Effects of video-game play on information processing: A metaanalytic investigation. Psychonomic Bulletin \& Review 20: 1055-1079. http://dx.doi.org/10.3758/s13423-013$0418-\mathrm{z}$

35. Kristopher J. Preacher, Derek D. Rucker, Robert C. MacCallum, W. Alan Nicewander. 2005. Use of the extreme groups approach: A critical reexamination and new recommendations. Psychological Methods 10, 2: 178-192. http://dx.doi.org/10.1037/1082-989X.10.2.178

36. Thomas S. Redick, James M. Broadway, Matt E. Meier, Princy S. Kuriakose, Nash Unsworth, Michael J. Kane, and Randall W. Engle. 2012. Measuring working memory capacity with automated complex span tasks. European Journal of Psychological Assessment 28, 3: 164-171. http://dx.doi.org/10.1027/1015-5759/a000123

37. Delphine Sasanguie, Silke M. Göbel, Kristina Moll, Karolien Smets, Bert Reynvoet. 2012. Approximate number sense, symbolic number processing, or numberspace mappings: What underlies mathematics achievement? Journal of Experimental Child Psychology 114, 3: 418-431. http://dx.doi.org/10.1016/j.jecp.2012.10.012

38. Nash Unsworth, Randall W. Engle. 2005. Working memory capacity and fluid abilities: Examining the correlation between Operation Span and Raven. Intelligence 33: 67-81.

http://dx.doi.org/10.1016/j.intell.2004.08.003

39. Nash Unsworth, Thomas S. Redick, Brittany D. McMillan, David Z. Hambrick, Michael J. Kane, Randall W. Engle. 2015. Is playing video games related to cognitive abilities? Psychological Science 26, 6: 759774. http://dx.doi.org/10.1177/0956797615570367

40. Alex Wilhelm. 2012. MLG's eSports events now attract more young male viewers than the Rose Bowl. Retrieved September 23, 2015 from http://thenextweb.com/media/2012/07/18/mlgs-esportsevents-now-attract-more-young-male-viewers-than-therose-bowl/

41. Nick Wingfield. 2014. In e-Sports, video gamers draw real crowds and big money. Retrieved September 23, 2015 from http://www.nytimes.com/2014/08/31/technology/esports -explosion-brings-opportunity-riches-for-videogamers.html?_r=0

42. Nick Yee. 2006. Motivations for play in online games. CyberPsychology \& Behavior 9, 6: 772-775. http://dx.doi.org/10.1089/cpb.2006.9.772 